\title{
Enhancement of the wettability of graphite-based lithium-ion battery anodes by selective laser surface modification using low energy nanosecond pulses
}

\author{
Max-Jonathan Kleefoot ${ }^{1,2} \cdot$ Sebastian Enderle ${ }^{1}$. Jens Sandherr ${ }^{3}$. Marius Bolsinger ${ }^{3}$. Thomas Maischik ${ }^{4}$. \\ Nadine Simon ${ }^{4} \cdot$ Jiři Martan $^{2} \cdot$ Simon Ruck $^{1} \cdot$ Volker Knoblauch $^{3} \cdot$ Harald Riegel $^{1}$
}

Received: 3 May 2021 / Accepted: 2 September 2021 / Published online: 16 September 2021

(C) The Author(s) 2021

\begin{abstract}
The electrolyte filling process of battery cells is one of the time-critical bottlenecks in cell production. Wetting is of particular importance here, since only completely wetted electrode sections are working. In order to accelerate and facilitate this process, the authors of this study developed a method to significantly increase the wettability of graphite-based anodes by a laser surface modification using low energy nanosecond laser pulses. The anode surface microstructure was evaluated by means of white-light interferometry and scanning electron microscopy. The assessment of wettability was done by drop test and capillary rise test of the liquid electrolyte. The results show that there is a predominantly selective ablation process for laser energy inputs below $2 \mathrm{~J} / \mathrm{m}$ by which the graphite active material remains unaffected and the binder material is decomposed. The observed increase in surface roughness correlates with the increasing wettability. Investigations using Raman spectroscopy showed that laser treatment leads to a damage on the crystalline structure of the graphite particle surface. However, treating an entire anode including $6 \mathrm{wt} \%$ binder and conductive carbon black has shown that the overall amorphous content of the anodes surface can be reduced by $32 \%$ through treating the surface with a laser energy of $1.29 \mathrm{~J} / \mathrm{m}$. Up to that point, which is the resulting parameter range for the selective process, it is possible to ablate the amorphous binder and carbon black phase coevally exposing graphite particles while keeping their crystalline structure. Exceeding that range, ablation of the whole anode composite dominates and amorphization of the graphite surface occurs. The electrode's capacity was tested on half-cells in coin cell format. For the whole laser parameter range investigated, the anodes capacity matches the mass loss caused by laser ablation. No additional capacity loss was observed due to amorphization of the exterior graphite particle's surface.
\end{abstract}

Keywords Wettability $\cdot$ Selective laser ablation $\cdot$ Short pulsed laser $\cdot$ Lithium-ion battery $\cdot$ Anode

\section{Introduction}

The time and cost-efficient production of Lithium-ion battery (LiB) cells is - besides realizing high energy and power densities - a crucial challenge for the establishment of LiBs as an

Max-Jonathan Kleefoot

Max-Jonathan.Kleefoot@hs-aalen.de

1 Laser Application Center, Aalen University, Beethovenstraße 1, D-73430 Aalen, Germany

2 New Technologies Research Centre (NTC), University of West Bohemia, Univerzitni 8, 30100 Plzen, Czech Republic

3 Materials Research Institute Aalen, Aalen University, Beethovenstraße 1, D-73430 Aalen, Germany

4 Volkswagen AG, Group Innovation, D-38440 Wolfsburg, Germany energy storage for future mobility solutions. During cell assembly, the battery cell which consists of a cathode, an anode and a separator in between the two electrodes are being filled with a liquid electrolyte. The filling process directly follows the assembly of the cell and its packaging in pouch bags, for example. The predominantly vacuum-assisted electrolyte filling process of these pouch bag cells is one of the major bottlenecks limiting the throughput of cell production. The homogeneous and complete soaking of the porous active mass layer of each electrode is essential for cell quality. An insufficient wetting might deteriorate cell performance and contribute to the formation of dendrites and thus cell damage [1-5].

The microstructural optimization of the electrode towards an enhanced wetting behavior is subject of current research [4, 6-9]. Besides mechanical and additive manufacturing methods, ablative laser material processing is applied for the creation of three- 
dimensional structures, such as grids, trenches, or a blind-holetype perforation $[4,7-10]$. Such micro-capillarities are capable of reducing the electrolyte filling time significantly. According to Pfleging et al. [8], using laser material processing to create threedimensional structures has an even more pronounced positive effect on the electrolyte wetting than mechanical forming processes. The authors conclude that this is a result of the surface porosity resulting from the polymer binder that is decomposed due to the thermal impact caused by the laser material interaction. The selective surface modification of cathodes that was presented by Ruck et al. [11, 12] apparently leads to a surface morphology comparable to the one of the capillarity sidewalls created by laser ablation in Pfleging and Pröll [8]. The work of Ruck et al. [11, 13] gave an insight into the mechanisms of this photothermal laser process. When the pulsed near infrared (NIR) laser radiation couples into the highly absorbing active mass of the electrode, heat is generated therein. As soon as the laser energy reaches a critical value, the polymeric binder begins to decompose and evaporates. As long as the process energy does not exceed a threshold value, the active material particles of the cathode are supposed to remain unaffected. The resulting surface shows an increased surface roughness, and thus, this process causes an enlargement of active surface area [11, 13].

In the present study, it was investigated to what extent the selective surface modification process presented by Ruck et al. $[11,12]$ is applicable on high energy graphite based anodes with a special focus on their wettability. To this end, the interaction of the laser with the different materials (graphite, polymeric binder, and carbon black as a conductive additive) used within the active mass of the anode in order to derive threshold values for the selective removal of the binder was elaborated. Using these parameters as a first approach for the laser treatment of anodes, in the second step, the effect of various laser parameters on the microstructural properties of the anode surface and the graphite itself was investigated. The laser machined anodes were analyzed using scanning electron microscopy (SEM), white-light interferometry (WLI), and Raman spectroscopy. Moreover, a gravimetric analysis to evaluate the mass removal and its effect on the cell capacity of differently machined electrodes was done. Last - in order to face the bottleneck during cell production - the effect of the selective laser treatment on the wettability of the anodes concerning liquid electrolyte was evaluated by a drop test as well as by measuring the rising height of the electrolyte in a specific test set-up.

\section{Experimental setup}

\subsection{Laser system}

The laser treatment was conducted with a TruMark5020 Yb:YAG Fiber laser of the company TRUMPF. Using a scanning optic with a focal length of $160 \mathrm{~mm}$, the laser beam with a wavelength of $1064 \mathrm{~nm}$ was focused on the top surface of the workpiece. The focal diameter was approximately $70 \mu \mathrm{m}$. In order to ensure a plane surface, the samples were fixed carefully onto a steel plate. All samples were treated with a pulse duration of $9 \mathrm{~ns}$ and a constant hatch distance of $35 \mu \mathrm{m}$. To investigate the influence of the laser treatment on the ablation depth and microstructure of the sample surface, the average laser power was varied from 0.9 to $4 \mathrm{~W}$, the scanning velocity from 0.5 up to $2.5 \mathrm{~m} / \mathrm{s}$, and thus the energy input per unit length $E_{\text {Dis }}=P_{\text {av }} / v_{\text {scan }}$ from 0.36 to $8 \mathrm{~J} / \mathrm{m}$. The pulse repetition rate was varied from 113.5 up to $200 \mathrm{kHz}$.

\subsection{Electrode material and electrochemical evaluation}

Anodes consisting of $94 \mathrm{wt} \%$ graphite (SGL carbon, synthetic graphite, D10 $=6.8 \mu \mathrm{m}, \mathrm{D} 50=18.4 \mu \mathrm{m}, \mathrm{D} 90=42.9 \mu \mathrm{m}$ ), 2 wt\% carbon black (Imerys, C-Nergy Super C65), 2 wt\% CMC (Nippon, Sunrose MAC 500LC), and $2 \mathrm{wt} \%$ SBR (Zeon, BM-451B) were used for the experiments.

Electrode slurry with a total solid content of $48 \mathrm{wt} \%$ (solved in water) was coated one-sided on a $10-\mu \mathrm{m}$-thick copper foil using the doctor blade method. Eventually, the graphite anodes had a mass loading of $9 \mathrm{mg} / \mathrm{cm}^{2}$ corresponding to an area capacity of $3 \mathrm{mAh} / \mathrm{cm}^{2}$ (assuming a gravimetric capacity of $362 \mathrm{mAh} / \mathrm{g}$ for the graphite). After drying overnight in ambient air at room temperature and subsequent calendaring, the coating had a thickness of $55 \mu \mathrm{m}$ corresponding to an active mass layer density of $1.6 \mathrm{~g} / \mathrm{cm}^{3}$. These samples were used throughout all following investigations. Only for the wetting tests, additional samples with a thickness of $64 \mu \mathrm{m}$ after calendaring and thus, a lower density of 1.4 $\mathrm{g} / \mathrm{cm}^{3}$, were produced.

The cell capacity was measured by building up half-cells consisting of a graphite anode with a diameter of $12 \mathrm{~mm}$ and a lithium foil (Sigma Aldrich, $750 \mu \mathrm{m}, 15 \mathrm{~mm}$ ) as counter electrode. The cells were assembled under argon atmosphere in CR2032 coin cell format. A Whatman Grade GF/A separator was used. The cells were filled with $150 \mu \mathrm{l}(1 \mathrm{M}$ LiPF6 in EC:DEC (3:7 wt \%) + $2 \%$ VC) electrolyte. A BaSyTec CTS-Lab battery tester was used for charging and discharging the cells three times with a current rate of $\mathrm{C} / 10$. The nominal (dis-)charge current, which is defining the current rate, was determined using the areal capacity of the anodes of $3 \mathrm{mAh} /$ $\mathrm{cm}^{2}$. That is resulting from gravimetric measurements of the electrodes and the nominal capacity of the graphite of 362 $\mathrm{mAh} / \mathrm{g}$.

\subsection{Microstructural and gravimetrical evaluation of the laser ablation}

The ablation depth and the surface roughness were evaluated using white-light interferometry (Zygo NewView 8300). Figure 1 visualizes the measurement principle of those surface 
properties. The average ablation depth $\Delta z$ was determined by measuring the difference between the mean height of an untreated reference area (right picture) and a laser treated area (left picture) next to it. Therefore, square white-light interferometry images with an edge length of $2 \mathrm{~mm}$ were captured in such way that half of the image covers the laser treated area and the other half covers the reference area. The change of the arithmetic mean roughness $\Delta S a$ was evaluated by taking separate square images with an edge size of $400 \mu \mathrm{m}$ of the untreated and the laser treated area. In the following, the difference in roughness between both areas is referred to as surface roughness $\Delta S a$. This difference is ascribed to the laser treatment. As stated by Enderle et al. [13] in the context of the laser treatment of cathodes, the surface roughness is appropriate for the evaluation of the laser induced changes of the electrode surface. However, in contrast to Habedank et al. [4], we utilized the arithmetic mean roughness $\mathrm{Sa}$ instead of the height of the roughness profile. Due to the wide distribution of the graphite flake size the $S a$-value allows a more representative evaluation of the surface properties.

In addition to that, top-view images of the anodes were taken with a Zeiss Leo Gemini 1525 scanning electron microscope (SEM) and cross-sectional images with a Zeiss Axio Imager Vario light microscope.

All gravimetric measurements were performed using an Ohaus AX324 weight scale. To obtain a representative gravimetric measurement result, samples with a size of $80 \times 80$ $\mathrm{mm}^{2}$ were measured before the laser treatment and afterwards. For these particular samples, the laser treated areas had a size of $70 \times 70 \mathrm{~mm}^{2}$.

The Raman spectroscopy was conducted using a Labram Aramis, Horiba Jobin Yvon GmbH. The data acquired covered a square area of $50 \times 50 \mu^{2}$. The mapping involves about 280 single measuring points. The wavelength of the laser is $632 \mathrm{~nm}$, and a $50 \times$ microscope objective was selected. The Raman analysis line was adjusted using a $155-\mu \mathrm{m}$ aperture and a $112-\mu \mathrm{m}$ slit; the diffraction grating number was selected to 1200 . The scanning range was $1000-1800 \mathrm{~cm}^{-1}$, the scanning time was set to $30 \mathrm{~s}$, and the number of scans was 3 . The Ramanshifts were analyzed using the LabSpec 5 software regarding the wavenumbers $1331 \mathrm{~cm}^{-1}$ (D-band) and $1580 \mathrm{~cm}^{-1}$ (G-band) being characteristic for the amorphous and crystalline character of carbon respectively [14]. In order to realize a quantitative comparison of the carbon character, the band-ratio $I_{G} / I_{D}$, given by the intensity $I$ of the two bands, was evaluated. Therefore LabSpec 5 provided the baseline correction, scaling and averaging of all mapping spectra of the individual pixels. Through deconvolution of the data, peak-fitting to 1331,1580 , and $1614 \mathrm{~cm}^{-1}$ bands was done using Gauss-Lorentz functions. Afterwards the peak intensities of the D-band and G-band were taken for $I_{G} / I_{D}$ band-ratio calculation. This determination of the band ratio is exemplarily visualized in Fig. 2 for carbon black. Therefore, the carbon black powder was compressed to a disk. The increase of the band ratio is proportional to the extent of the crystalline character of the carbon. In contrast to the graphite flakes used as active material, the carbon black contained within the binder compound has a low $I_{G} / I_{D}$-ratio $<1$, hence a predominantly amorphous character (see Fig. 2).

The verification of the Raman measurements on anodes was done by analyzing identically treated pure crystalline graphite discs consisting of the compressed active material. These discs had a thickness of approximately $4 \mathrm{~mm}$ and a diameter of $25 \mathrm{~mm}$.

\subsection{Analysis of the wetting behavior}

The wettability of the anode surface with the liquid electrolyte was evaluated regarding two different use cases. First, we placed a defined drop of $5 \mu \mathrm{l}$ electrolyte in the middle on top of the anode samples. It was taken care that the sample surface was even and horizontal. After $120 \mathrm{~s}$, top-view photos of the sample surface were captured with a digital camera. Afterwards, the area that has been wetted with electrolyte
Fig. 1 Measurement principle of the average ablation depth $\Delta z$ and the change of surface roughness $\Delta \mathrm{Sa}$

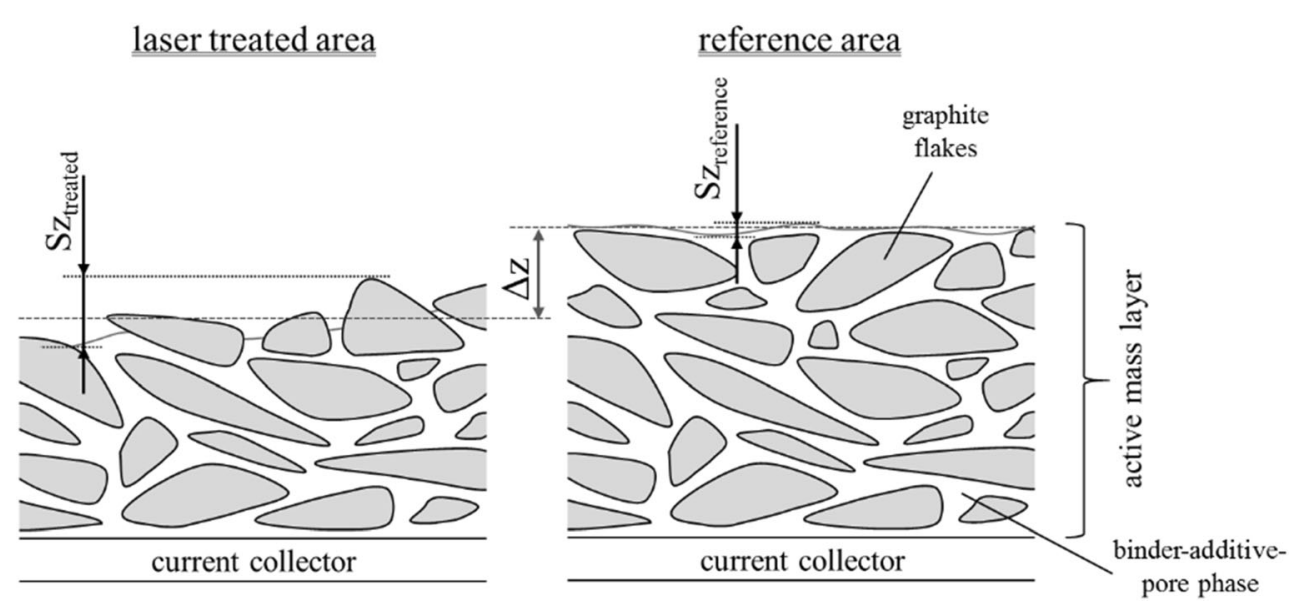


Fig. 2 Raman spectroscopy results of the $I_{G} / I_{D}$-band ratio determination for carbon black

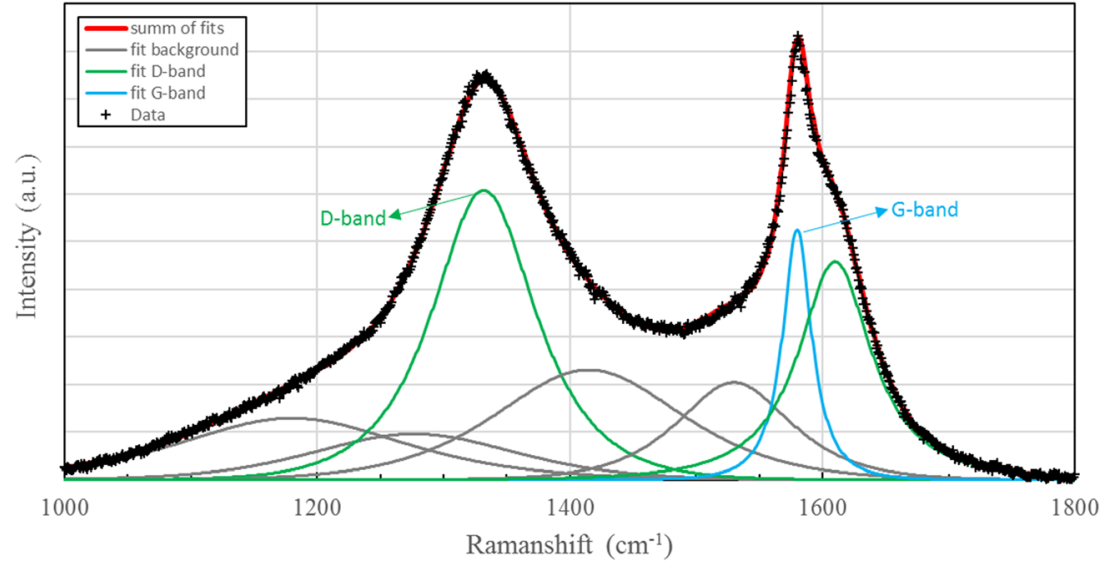

within $120 \mathrm{~s}$ was determined by counting the pixels of the photo being visually changed due to the wetting process. Figure 3 a illustrates the test principle.

The evaluation of the wetting behavior in a more application orientated manner was done by electrolyte rising tests. This approach considers the through-thickness soaking of the entire active mass layer as it gains importance during the electrolyte filling process in cell production. Therefore, the rise of electrolyte within vertically mounted anode samples was analyzed. As illustrated in Figure 3b, the samples were fixed on microscope slides and dipped into an electrolyte bath. After $10 \mathrm{~min}$, the rising height was measured with a millimeter-scale. The initial surface of the electrolyte bath was used as base line and equals a rising height of zero.

To determine the waiting times, a preliminary study was carried out in which the wetting evolution over time was analyzed. In case of the drop-test, the point in time at which no significant change in the wetting area can be observed was decisive, being reached after $120 \mathrm{~s}$. For the rising height test, the rising electrolyte approaches a limit after approximately 10 minutes.

\section{Results and discussion}

\subsection{Laser process development and ablation mechanisms}

One approach to achieve a selective ablation process bases on the different ablation thresholds of the single workpiece components. In the following, we will therefore distinguish between the active material consisting of the comparably large graphite particles and the binder-additive compound consisting of CMC, SBR, and carbon black powder. The latter ought to be ablated and the former ought to be unaffected by the laser radiation and its thermal impact within the workpiece.

Figure 4 depicts the results of ablation threshold tests for the two anode components. Therein, the intersection of both fitting lines and the abscissa represent the ablation threshold of each anode component. It can be seen that the ablation thresholds of both anode components differ significantly. Derived from the figure, using the given laser setup, the active material has an ablation threshold of approximately $2 \mathrm{~J} / \mathrm{m}$, whereas the binder additive compound is already being ablated at approx.
Fig. 3 Measurement of the wetted area during drop-wetting test (a) and the capillary rise of the electrolyte (b)

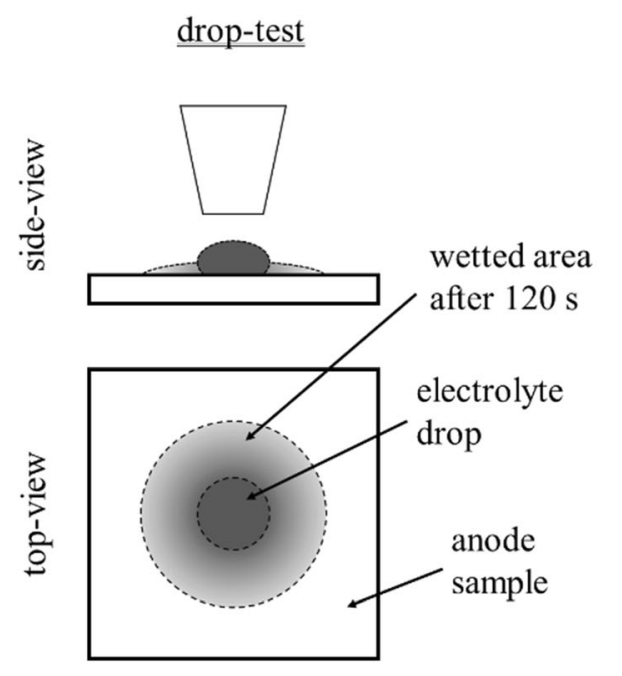

a) $\underline{\text { rising height test }}$

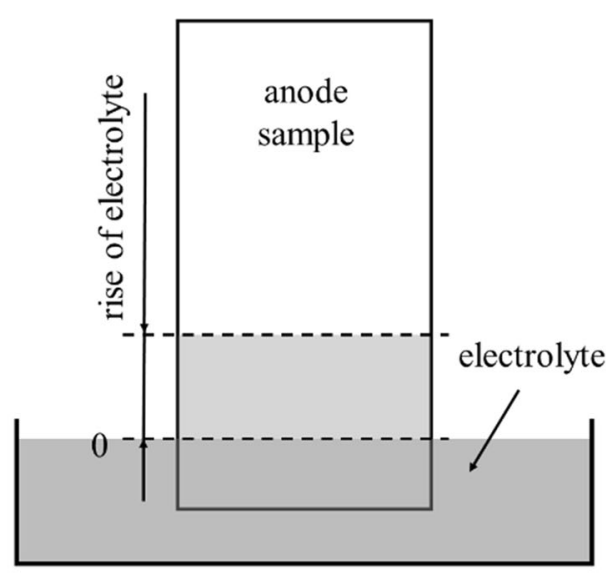

b) 


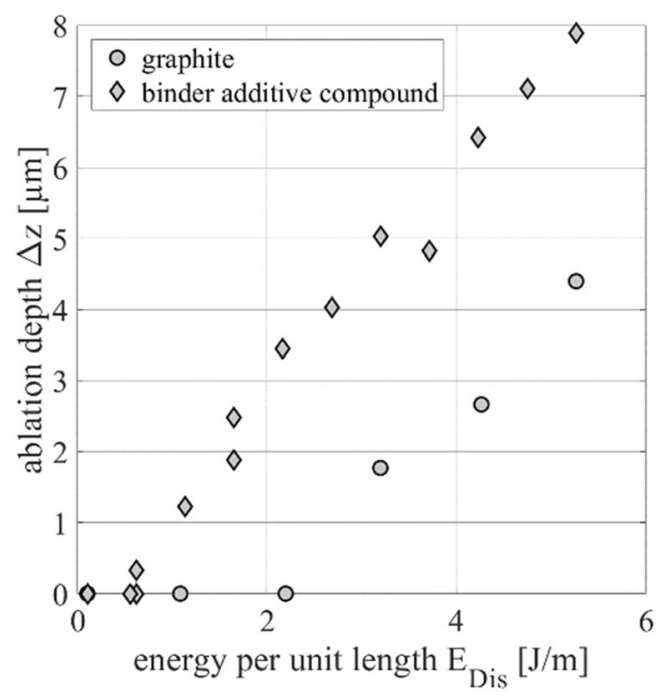

Fig. 4 Ablation threshold of active material and binder additive compound using a constant pulse repetition rate of $200 \mathrm{kHz}$

$0.8 \mathrm{~J} / \mathrm{m}$. This circumstance allows to define the range between the two ablation thresholds as a promising process window for the selective ablation of the binder additive compound.

Figure 5 depicts the results of the average ablation depth $\Delta z$ on the left and the laser-induced change of the surface roughness $\Delta S a$ on the right ordinate axis, both as a function of energy input per unit length. The results show that the ablation depth and the surface roughness strongly correlate with the energy input. This also agrees with the findings in literature concerning the selective ablation process applied on cathodes $[11,13]$. Having a closer look, it becomes obvious that for an energy input below $2 \mathrm{~J} / \mathrm{m}$, only a negligible ablation depth occurs. Exceeding this apparent threshold energy input causes a significant material removal which almost increases linearly along with the energy input per unit length. This threshold energy input is in good agreement with the ablation threshold previously derived for the single active material.
Meanwhile, the surface roughness is increasing along with the laser energy as well. Similar to the ablation threshold tests, the curve of the surface roughness is shifted towards lower energy inputs in such extend that the intersection of the curve with the abscissa is within an energy range comparable to the ablation threshold of the binder additive compound. This might indicate a transition between a primarily selective ablation of the binder additive compound for energy inputs between approximately $0.8 \mathrm{~J} / \mathrm{m}$ and $2 \mathrm{~J} / \mathrm{m}$ and a process regime $(>2 \mathrm{~J} / \mathrm{m})$ in which also active material particles are being removed. However, the active material particles are embedded within the binder additive compound. Thus, it cannot be clarified, whether the active material is removed due to the vapor dynamics of the decomposed binder or due direct ablation of the graphite itself. Most likely, the former case will occur for lower energy inputs whereas the latter will arise for high energy inputs. Note, that for higher energy inputs $(>5 \mathrm{~J} / \mathrm{m})$, the increase in surface roughness seems to flatten out. This flattening and the strong increase of ablation depth in the same energy range might be resulting from the dominance of the directly ablated active material. Thus, a layer-wise removal of the active mass might take place, including the decomposition of the binder and the ablation of the active material. Furthermore, the fact that both evaluated properties correlate with the energy input per unit length substantiates the thermal process principle as it is described in Enderle et al. [13].

In Fig. 6, both measurands are opposed to each other. The laser induced change of the surface roughness is given as a function of the average ablation depth. Especially for lower ablation depths, the surface roughness shows a steep incline. From an ablation depth of approximately $6 \mu \mathrm{m}$ onwards the curve flattens-out as well, indicating that ablation takes place without remarkable change of the surface roughness. Furthermore, the gradient of the curve seems to reach an approximately constant value. As already reported in literature
Fig. 5 Average ablation depth and laser-induced change of surface roughness as a function of energy per unit length

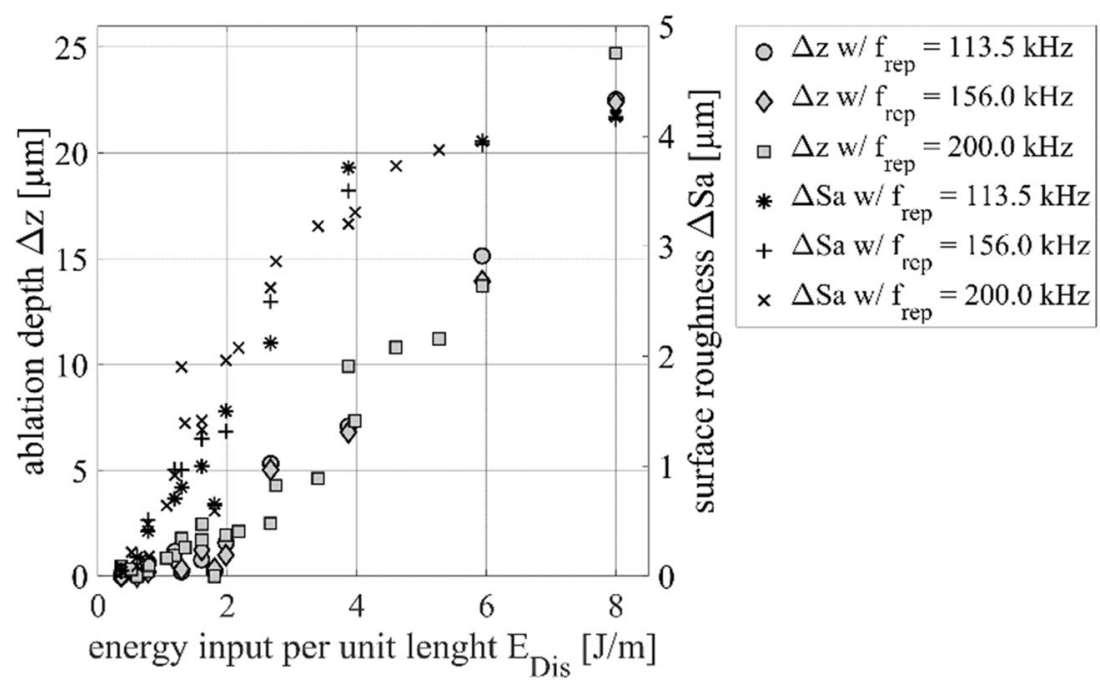




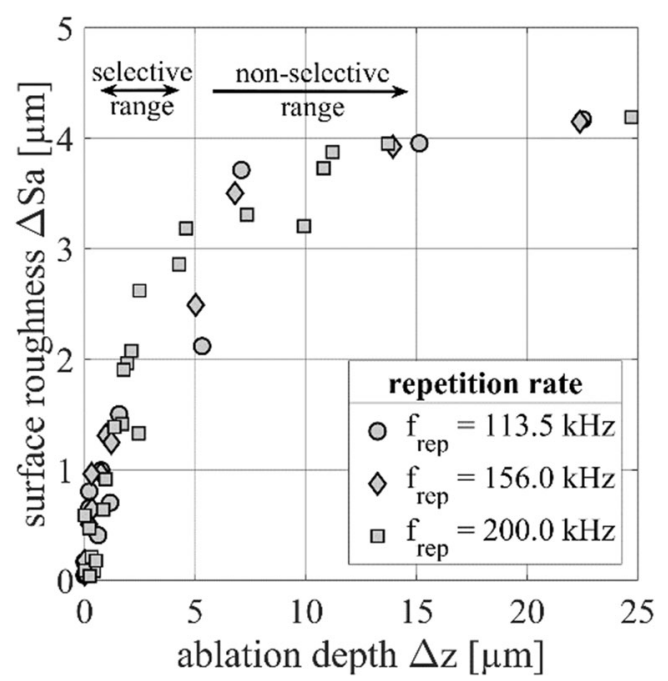

Fig. 6 Laser-induced change of surface roughness as a function of ablation depth

$[11,13]$ and described earlier, this progression of the curve might be ascribed to a transition from a process regime that is dominated by the selective removal of certain workpiece components such as the binder additive compound to a nonselective ablation regime.

To investigate this transition and to gain more information about materials and microstructural changes due to the laser treatment, four specific laser parameters (see Table 1) were picked and detailed analyses by SEM and Ramanspectroscopy were carried out.

Top-view and cross-sectional SEM images of these anodes are compared in Fig. 7 with an untreated reference anode. The SEM images substantiate the conclusions drawn from roughness and ablation depth measurements discussed above. The gray lines in the cross-sectional images (lower row of images) were drawn manually in order to facilitate the differentiation between the anode surface and the binder-like looking embedding resin. The cross-sectional images of the samples treated with parameter (I) and (II) confirm the negligible ablation depth in this range of energy input per unit length. However, the top-view images of these samples (upper row of images) show an increase of surface pores and suggest an overall increase of the surface roughness as measured by white-light interferometry. Apart from that, no removal of graphite particles could be discovered for these parameters. Exceeding the threshold of approximately $2 \mathrm{~J} / \mathrm{m}$ (parameters (III) and (IV)), a more pronounced roughening of the surface as well as a measurable reduction of the electrode thickness can be observed. This reduction goes along with the increase of the average ablation depth measured for these parameters and the assumption of a process influenced by a non-selective removal of the entire active mass.

The transition from a process regime dominated by a bare roughening of the surface towards a removal of the entire active mass also matches the findings in our previous study [13]. However, due to the wide size distribution of the graphite particles that can be seen in the SEM images, it seems to be likely that there is more or less a continuous transition from the removal of the binder-additive compound that was identified to be in the range of $2 \mathrm{~J} / \mathrm{m}$, towards the concurrent removal of the graphite particles - instead of a distinct transition between both regimes. This assumption is supported by the wide distributed size of the graphite particles. Thus, small graphite particles are removed together with the thermal decomposed binder, whereas bigger sized particles would remain almost unaffected by the ablation process.

\subsection{Influence of laser parameters on the crystalline structure of the graphite}

To investigate the impact of the laser treatment on the crystalline graphite structure, Raman spectroscopy has been carried out utilizing the pure graphite discs as well as calendered anodes that have been treated with various laser energy inputs (laser parameters (I) to (IV)). Figure 8 summarizes the results by plotting the band ratio $I_{G} / I_{D}$ which serves as a measure for the crystallinity of the analyzed material. In the left figure, the band ratios for each laser parameter are depicted. Therein, the results concerning the anode samples are represented by the circles, those of the graphite discs by the asterisks, respectively. The image in the left bottom corner visualizes the region of interest $\left(50 \times 50 \mu^{2}\right.$, colored in cyan) for the measurements. On the right half of the figure, the areal distribution of the band ratio for anodes (upper row) and graphite discs (lower row) are exemplarily depicted for the untreated reference sample, parameter (II) and parameter (IV).

Compared to the untreated reference sample, the measured band ratio of samples treated with (I) and (II) suggest an increase of the crystalline character of the active mass compound due to the interaction with the laser. Applying higher
Table 1 Selected laser parameters for detailed investigations on the anode microstructure

\begin{tabular}{llll}
\hline Parameter & Average laser power (W) & Scanning speed $(\mathrm{m} / \mathrm{s})$ & Energy input per unit length $(\mathrm{J} / \mathrm{m})$ \\
\hline (I) & 0.41 & 1.5 & 0.62 \\
(II) & 0.86 & 1.5 & 1.29 \\
(III) & 1.44 & 1.5 & 2.16 \\
(IV) & 1.78 & 1.5 & 2.67 \\
\hline
\end{tabular}




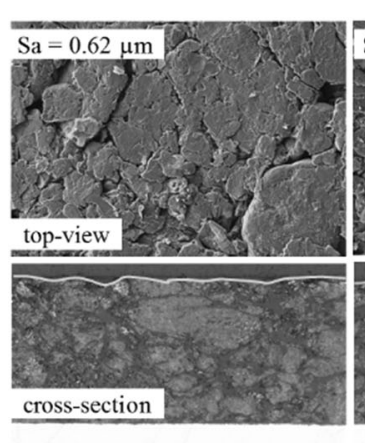

(reference)

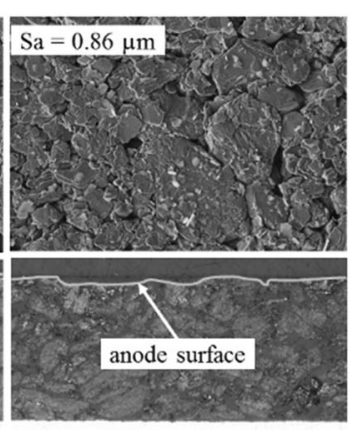

(I)

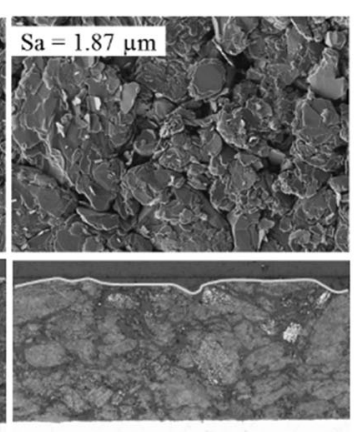

(II)

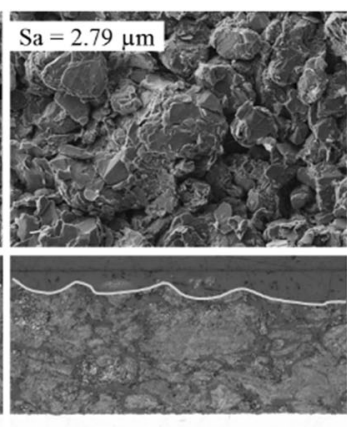

(III)

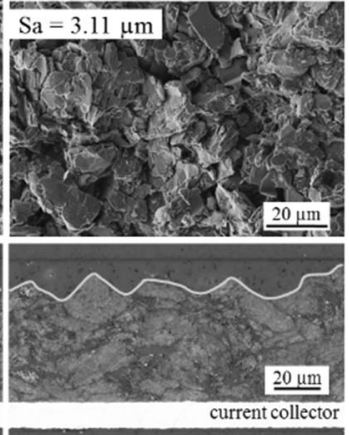

(IV)
Fig. 7 Top-view SEM images of anode samples treated with various energy inputs per unit length in the top row and cross-sectional light microscope images of the identically treated samples in the bottom row.
The white lines were added manually to visualize the border between the active mass layer and the embedding liquid laser energy inputs with (III) and (IV), the band ratio decreases below the reference value, indicating a decrease of the crystallinity of the material compound. In contrast, an ongoing amorphization is observed for the pure graphite. This is suggested by a continuous decrease of the band ratio from 5.1 to 1. These findings can be interpreted as follows and in turn support the hypothesis of two different ablation regimes: For low energy inputs, the predominantly amorphous binderadditive compound is removed selectively from the active mass (note that the $I_{G} / I_{D}$-ratio of pure carbon black is only 1.1 (see Fig. 2) indicating its amorphous character), resulting in an increase of the crystalline character of the remaining material compound. Admittedly, the graphite starts to get amorphous in this regime as well, but we assume that this effect is overcompensated by the ablation of the amorphous carbon black and thus, an overall increase of the crystallinity can be detected. With higher energy inputs ((III) and (IV)), the ongoing amorphization of the graphite becomes dominant resulting in an overall decreasing band-ratio of the active material compound.

The presented laser ablation process strives for the avoidance of any negative impact on the active material and thus the electrochemical performance of the battery cell. Besides the removal of the active material, any microstructural changes of the same such as an amorphization are undesirable. Hence, the results of the Raman spectroscopy support the presumption that parameter (II) covers a promising range for the energy input per unit length of the selective ablation process. With regard to the application of this process in battery production, it seems to be preferable to avoid the removal of active material, since this results in a reduction of electrodes capacity. Therefore, the investigations presented in this work aim for a maximized roughening of the surface - and thus, for an increase of surface porosity and the enhancement of capillary effects - while keeping the removal of the active material at a minimum.

\subsection{Wetting behavior}

In the following, we present the impact of the described microstructural changes on the wettability of the anodes with liquid electrolyte during drop wetting and electrolyte rising test. With the latter, we intend to simulate the situation during electrolyte filling of a cell where the access of the electrolyte is
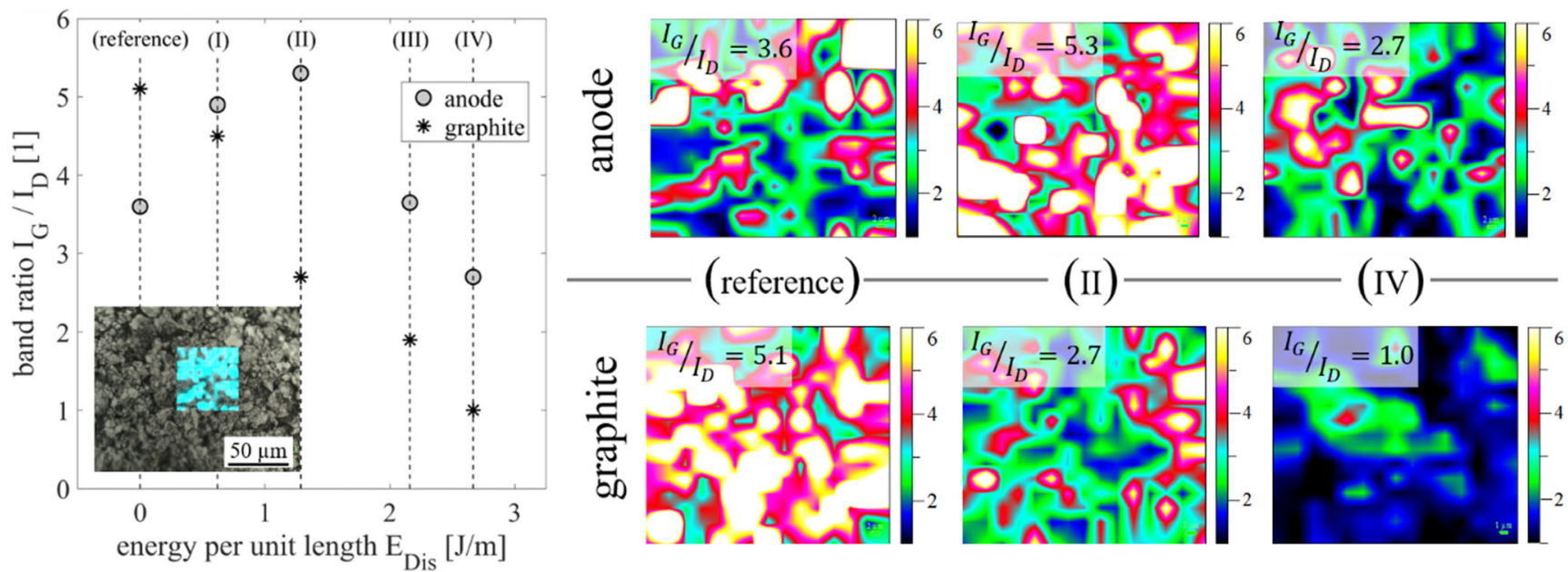

(II)
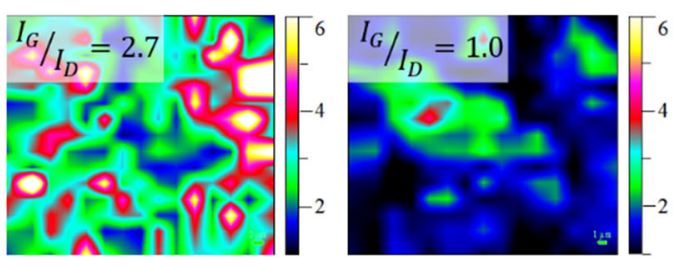

Fig. 8 Results of the Raman spectroscopy of anode samples that were treated with preselected laser parameters 
limited to the edges of the electrodes, respectively the jelly roll or the electrode stack. In general the surface modification leads to significant enhancement of the wetting behavior, comparable to the three-dimensional structuring of electrodes presented by Pfleging et al. [9].

The top view pictures taken $120 \mathrm{~s}$ after the electrolyte drop was placed on the surface are shown in Fig. 9. The numbers given in each picture quantify the relative increase of the wetted area, defined by the ratio of the wetted area of the laser treated sample $A_{t}$ and the wetted area of the untreated reference $A_{\text {ref }}$. Both anode types, the moderately densified anode with a density of $1.4 \mathrm{~g} / \mathrm{cm}^{3}$ as well as the higher densified anode with $1.6 \mathrm{~g} / \mathrm{cm}^{3}$, show a remarkable increase of the wetted area due to the laser treatment. Similar to the surface roughness, the enlargement of the wetted area increases along with the energy input per unit length. Especially the $1.6 \mathrm{~g} / \mathrm{cm}^{3}$ samples with a very smooth and plane surface after calendaring $(S a=0.33 \mu \mathrm{m})$ show a rather weak wettability. Hence, the laser-induced surface roughening ((II)-(IV)) leads to an even more pronounced increase of the wetted area up to a factor of 8. Vice versa, for moderately densified anodes $(S a=1.00 \mu \mathrm{m})$, the effect of the laser treatment is not as high - but still the wetted areas of the samples treated with parameters II, III, and IV are about three times larger compared to the untreated reference.

Figure 10 displays photos of the samples being dipped into electrolyte after $10 \mathrm{~min}$. The numbers given in this picture quantify the relative increase of the electrolyte rising height, defined by the ratio of the wetted height of the laser treated sample $S_{t}$ and the wetted height of the untreated reference $S_{\text {ref. }}$. Applying laser parameter (I) negligibly increases the rise of electrolyte by the factor 1.11 for $1.4 \mathrm{~g} / \mathrm{cm}^{3}$ and 1.34 for 1.6 $\mathrm{g} / \mathrm{cm}^{3}$ samples, respectively. A strong effect on the electrolyte rise is observed for a laser energy input of $\geq 2.16 \mathrm{~J} / \mathrm{m}$, i.e., the rising height increases up to the factor 3.71 for $1.4 \mathrm{~g} / \mathrm{cm}^{3}$
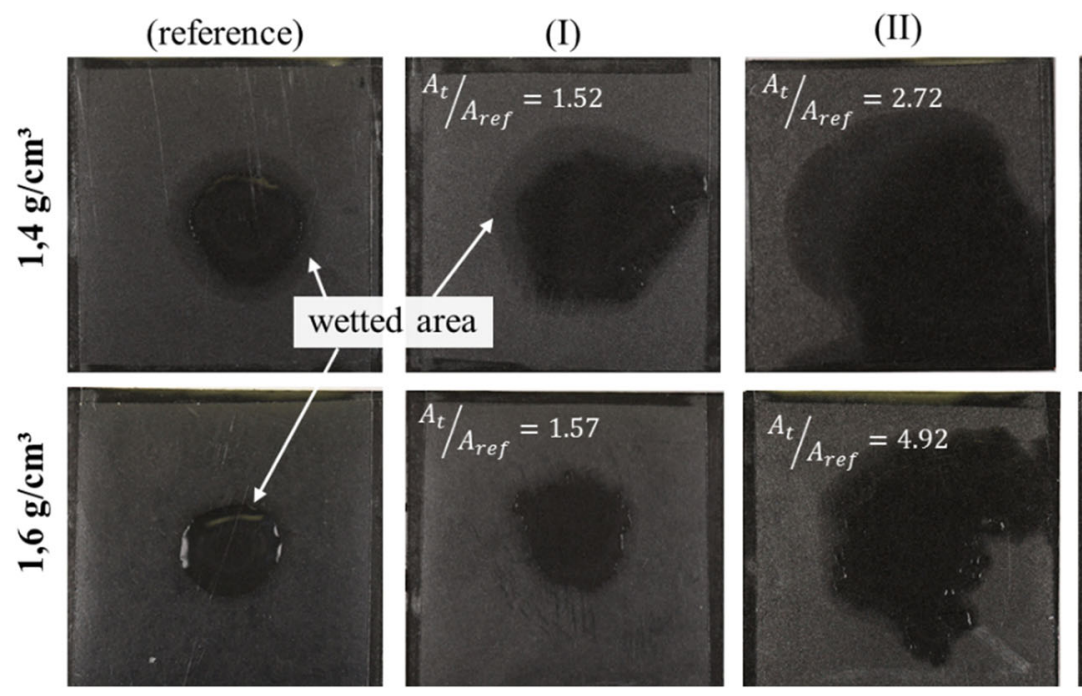

samples and 4.91 for $1.6 \mathrm{~g} / \mathrm{cm}^{3}$ samples. Again, the effect is more pronounced for the higher densified anodes. However, the improvement of wettability observed with this test is not as high as with the drop test. In contrast to the drop test which is dominated by the surface properties, the rising height determined with the rising height test is strongly influenced by the porosity over the entire active mass thickness - which itself is not affected by the superficial laser treatment. Nevertheless, both tests reveal a significant improvement of the wettability which in turn gives hope for an improved, i.e., faster and more homogeneous electrolyte filling in cell production.

\subsection{Electrode capacity}

The loss of active material that was observed during the gravimetrical and microstructural analysis is expected to influence the electrode capacity accordingly. In order to get an insight into the effect of the laser treatment on the electrode capacity, tests with coin cells (CR2032 format) were conducted. Figure 11 depicts the mean lithiation capacities of three identically treated cells and their standard deviations, which were measured during the first three charging and discharging cycles at a current rate of $\mathrm{C} / 10$.

The results after cell formation match the findings reported so far: During the first cycle, all samples exhibit an irreversible capacity loss which is well known and attributed to the formation of the solid electrolyte interface (SEI) [15]. After formation (3rd cycle) samples treated with laser parameter (I) do not differ significantly from the untreated reference sample which reached a capacity of $3.03 \mathrm{mAh} / \mathrm{cm}^{2}$. This value is in good agreement with the calculated areal capacity of 3.06 $\mathrm{mAh} / \mathrm{cm}^{2}$ being derived from the mass loading of $9 \mathrm{mg} / \mathrm{cm}^{2}$ and the nominal capacity of the graphite which was used for the electrode of $362 \mathrm{mAh} / \mathrm{g}$. Laser treatment with (III) and (IV) causes a pronounced capacity loss of $0.2 \mathrm{mAh} / \mathrm{cm}^{2}$ and

Fig. 9 Top-view images of the wetted anode surface exposed to a drop of electrolyte (after 120 seconds) 


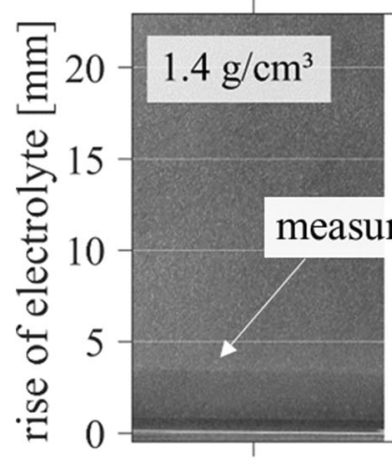

(reference)

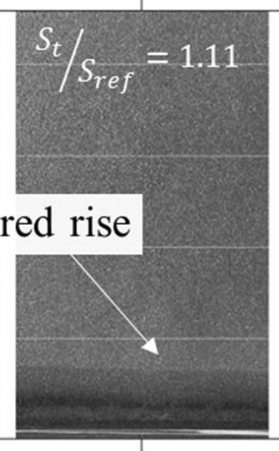

(I)

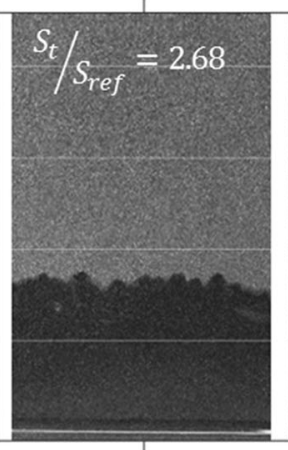

(II)

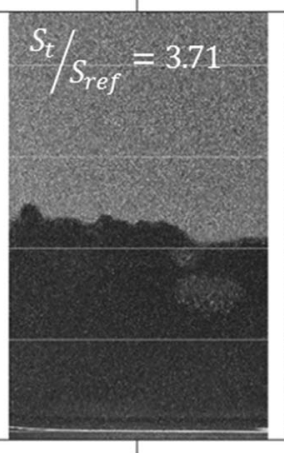

(III)

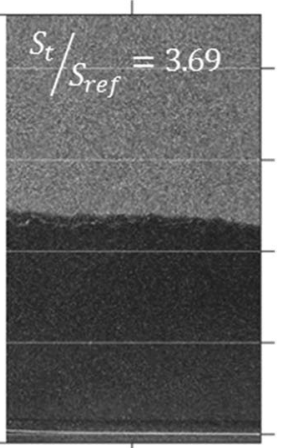

(IV)
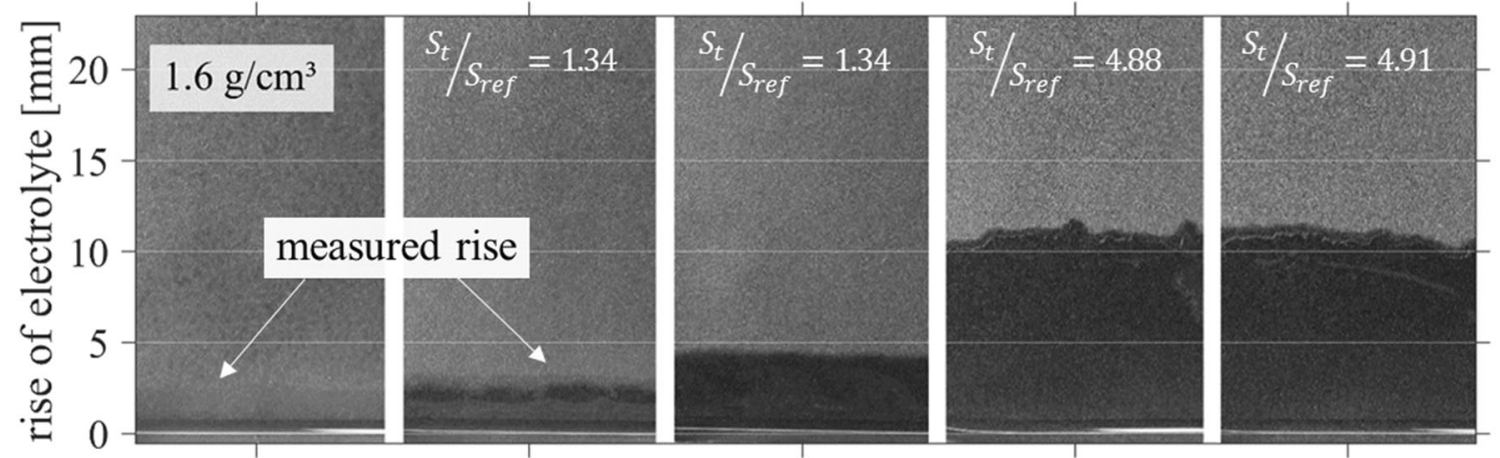

Fig. 10 Capillary rise of electrolyte on anode samples treated with the preselected laser parameters

$0.26 \mathrm{Ah} / \mathrm{cm}^{2}$ (corresponding to $6.6 \%$ and $8.58 \%$ ) whereas laser treatment with (II) results in a minor decrease of the lithiation capacity of about $0.05 \mathrm{mAh} / \mathrm{cm}^{2}$ (corresponding to $1.65 \%)$. As shown in Table 2, the calculated cell capacity after laser treatment based on the mass loss are matching the results of the experimentally determined lithiation capacities in Fig. 11.

This substantiates the interpretation of two different process regimes: The first - occurring at low laser energy inputs — is dominated by a selective removal of mostly inactive

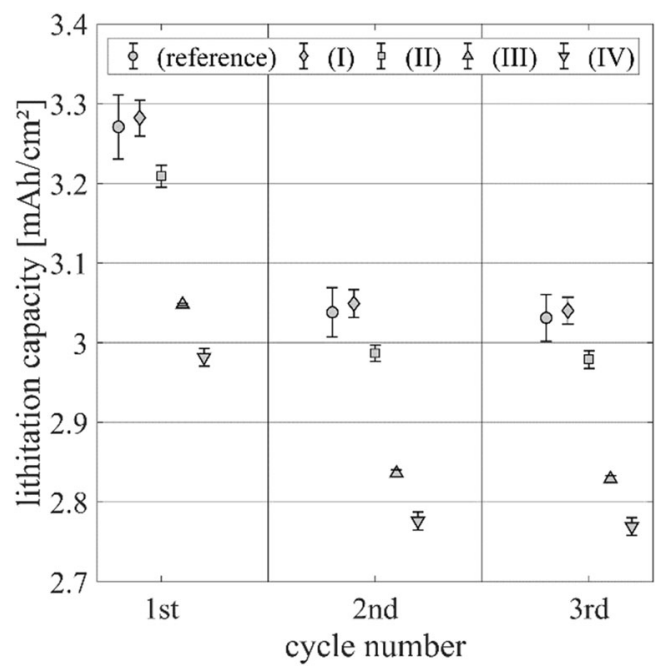

Fig. 11 Real lithiation capacities measured in half-cells in coin cell format electrode material such as the binder-additive compound and minor active material particles. The second - occurring at higher energy inputs - being dominated by the removal of the entire active mass compound. Especially the latter regime affects cell capacity. Considering the fact, that the experimentally determined capacities are slightly below the calculated values, the question arises whether this difference is caused by the amorphization of the graphite, that was observed by Raman spectroscopy. However, the differences are in the range of $1 \%$ and thus, low compared with the standard deviations of each cell. In addition, the differences are independent of the energy input of the laser treatment. Therefore they do not correlate with the degree of graphite amorphization. It might be concluded that — as far as low current rates are concerned - a possible negative impact of the active material amorphization on the electrode capacity is negligible compared with the capacity loss due to the laser induced removal of active material.

Of course, these capacity tests only give a first insight into the impact of the laser-based surface treatment of graphite anodes on their electrochemical performance. In order to achieve a comprehensive understanding, detailed electrochemical characterizations of laser treated anodes such as electrochemical impedance spectroscopy and rate capability tests are obligatory. However, these would go beyond the scope of the present study which covers the laser process development, the ablation processes and the effect of the laser processing on the wettability of the electrodes. 
Table 2 Results of the gravimetric and electrochemical analysis of coin cell half-cells

\begin{tabular}{llllll}
\hline Parameter & $\begin{array}{l}\text { Mass loss } \\
\left(\mathrm{mg} / \mathrm{cm}^{2}\right)\end{array}$ & $\begin{array}{l}\text { Calculated areal capacity } \\
\text { based on mass loss } \\
\left(\mathrm{mAh} / \mathrm{cm}^{2}\right)\end{array}$ & $\begin{array}{l}\text { Real cell capacity after } \\
\text { lithiation }(3 \mathrm{rd} \mathrm{cycle} \mathrm{with} \\
\mathrm{C} / 10)\left(\mathrm{mAh} / \mathrm{cm}^{2}\right)\end{array}$ & $\begin{array}{l}\text { Absolute capacity loss after } \\
\text { lithiation }(3 \mathrm{rd} \text { cycle with C/10) } \\
\left(\mathrm{mAh} / \mathrm{cm}^{2}\right)\end{array}$ & $\begin{array}{l}\text { Relative capacity loss after } \\
\text { lithiation }(3 \mathrm{rd} \mathrm{cycle} \mathrm{with} \\
\mathrm{C} / 10)(\%)\end{array}$ \\
\hline (reference) & 0.00 & 3.06 & 3.03 & - & - \\
(I) & 0.02 & 3.05 & 3.04 & -0.01 & -0.33 \\
(II) & 0.19 & 3.00 & 2.98 & 0.05 & 1.65 \\
(III) & 0.58 & 2.86 & 2.83 & 0.20 & 6.60 \\
(IV) & 0.73 & 2.81 & 2.77 & 0.26 & 8.58 \\
\hline
\end{tabular}

\section{Conclusion}

The above presented investigations give an overview on the effect of various laser parameters on the anode microstructure and the potential of the selective laser ablation process to enhance the wetting behavior of graphite-based anodes. Conducting ablation tests, the applicability of this process was investigated. Subsequently the laser-induced change of the microstructure was analyzed using scanning electron microscopy and Raman spectroscopy. Gravimetric and electrochemical investigations were conducted to get a first impression of the impact of the laser treatment on the cell capacity. The major conclusions drawn from these investigations can be summarized as follows:

- The laser treatment leads to a roughening of the anode surface. For lower energy $(<2 \mathrm{~J} / \mathrm{m})$ inputs the extent of the roughening is proportional to the energy input per unit length and mostly independent of the pulse repetition rate. A further increase of the laser energy input does not cause any further increase in roughness.

- The investigated process range can be divided into two regimes. One regime at low energy inputs is dominated by the selective removal of the binder-additive compound due to its thermal decomposition. The other one beginning at energy inputs above the ablation threshold of the active material is dominated by the ablation of the entire active mass. Note that there is no distinct step between both regimes but a continuous transition within a certain range.

- The cell capacity is significantly reduced when the active mass, i.e., including graphite particles is removed. The results of the capacity measurements are in good agreement with the calculated cell capacities derived from gravimetric measurements. Even though Raman spectroscopy indicates that the graphite is partially amorphized, a negative effect on the electrodes capacity due to that could not be observed in first electrochemical investigations. However, this needs more detailed investigations e.g. by electrochemical impedance spectroscopy.

- Electrolyte wetting tests demonstrate that the surface roughening that goes along with the laser treatment remarkably improves the wettability of the electrodes. Both tests that have been conducted - droplet-wetting and a capillary rise test - reveal a significant improvement of the wettability.

- Since the selective laser ablation process has been approved of having a significant positive impact on the fast-charging abilities of cathodes [12], its impact on the electrochemical performance of correspondingly treated anodes is worth further investigations.

Author contribution Max-Jonathan Kleefoot and Sebastian Enderle contributed to the conception of the study, performed the ablation experiment, the analysis, and the manuscript preparation; Jens Sandherr and Marius Bolsinger contributed to the cell-testing and wetting experiments including the analysis; Thomas Maischik and Nadine Simon contributed to the Raman spectroscopy and the analysis; Jiři Martan, Simon Ruck, Volker Knoblauch, and Harald Riegel helped perform the analysis with constructive discussions

Funding Open Access funding enabled and organized by Projekt DEAL. This work was supported by the German Federal Ministry of Economic Affairs and Energy by funding the project "structur.E - Strukturierte Anoden für verbesserte Schnellladefähigkeit und Steigerung der Energiedichte von Lithium-Ionenbatterien" (No. 03ETE018F).

Data availability The datasets used in the study are available on reasonable request from the corresponding author.

\section{Declarations}

Ethical approval Not applicable.

Consent to participate Not applicable.

Consent for publication Not applicable

Competing Interest The authors declare no competing interests.

Open Access This article is licensed under a Creative Commons Attribution 4.0 International License, which permits use, sharing, adaptation, distribution and reproduction in any medium or format, as long as you give appropriate credit to the original author(s) and the source, provide a link to the Creative Commons licence, and indicate if changes were made. The images or other third party material in this article are included in the article's Creative Commons licence, unless indicated otherwise in a 
credit line to the material. If material is not included in the article's Creative Commons licence and your intended use is not permitted by statutory regulation or exceeds the permitted use, you will need to obtain permission directly from the copyright holder. To view a copy of this licence, visit http://creativecommons.org/licenses/by/4.0/.

\section{References}

1. Korthauer R (2013) Handbuch lithium-ionen-batterien. Springer Vieweg, Berlin Heidelberg

2. Knoche T, Surek F, Reinhart G (2016) A process model for the electrolyte filling of lithium-ion batteries. Proc CIRP 41:405-410

3. Wood DL, Li J, Daniel C (2015) Prospects for reducing the processing cost of lithium ion batteries. J Power Sourc 275:234-242

4. Habedank JB, Günter FJ, Billot N, Gilles R, Neuwirth T, Reinhart G, Zaeh MF (2019) Rapid electrolyte wetting of lithium-ion batteries containing laser structured electrodes: in situ visualization by neutron radiography. Int J Adv Manuf Technol 102(9-12):27692778

5. Sauter C, Zahn R, Wood V (2020) Understanding electrolyte infilling of lithium ion batteries. J Electrochem Soc 167(10): 100546

6. Sheng Y, Fell CR, Son YK, Metz BM, Jiang J, Church BC (2014) Effect of calendering on electrode wettability in lithium-ion batteries. Front Energy Res 2:83

7. Pröll J, Kohler R, Adelhelm C, Bruns M, Torge M, Heißler S et al (2011) Laser modification and characterization of Li-Mn-O thin film cathodes for lithium-ion batteries. In: Laser-based Micro- and Nanopackaging and Assembly V: SPIE, 79210Q
8. Pfleging W, Pröll J (2014) A new approach for rapid electrolyte wetting in tape cast electrodes for lithium-ion batteries. J Mater Chem A 2(36):14918-14926

9. Pfleging W (2021) Recent progress in laser texturing of battery materials: a review of tuning electrochemical performances, related material development, and prospects for large-scale manufacturing. Int J Extrem Manuf 3(1):12002

10. Ferrari S, Loveridge M, Beattie SD, Jahn M, Dashwood RJ, Bhagat $\mathrm{R}$ (2015) Latest advances in the manufacturing of 3D rechargeable lithium microbatteries. J Power Sourc 286:25-46

11. Ruck S, Enderle S, Bolsinger M, Weller M, Knoblauch V, Riegel H (2019) Enhancing the rate capability of highly densified Li-Ion battery cathodes by selective laser ablation. In: Klotzbach U, Kling R, Watanabe A, editors. Laser-based Micro- and Nanoprocessing XIII: SPIE; 2019 - 2019, p 47

12. Bolsinger M, Weller M, Ruck S, Kaya P, Riegel H, Knoblauch V (2020) Selective surface treatment by means of IR-laser - a new approach to enhance the rate capability of cathodes for Li-ion batteries. Electrochim Acta 330:135163

13. Enderle S, Bolsinger M, Ruck S, Knoblauch V, Riegel H (2020) Thermophysical modeling of selective laser ablation processing of lithium-ion battery cathodes. J Laser Appl 32(4):42008

14. Baddour-Hadjean R, Pereira-Ramos J-P (2010) Raman microspectrometry applied to the study of electrode materials for lithium batteries. Chem Rev 110(3):1278-1319

15. Verma P, Maire P, Novák P (2010) A review of the features and analyses of the solid electrolyte interphase in Li-ion batteries. Electrochim Acta 55(22):6332-6341

Publisher's note Springer Nature remains neutral with regard to jurisdictional claims in published maps and institutional affiliations. 